\title{
On the influence of submarine ridges on translation and stability of Agulhas rings
}

\author{
Jens-Olaf Beismann, ${ }^{1}$ Rolf H. Käse, and Johann R. E. Lutjeharms ${ }^{2}$ \\ Institut für Meereskunde an der Universität Kiel, Kiel, Germany
}

\begin{abstract}
A series of experiments with a quasi-geostrophic model have been carried out to investigate the influence of topographic obstacles on the translatory movement of Agulhas rings. The rings were initialized as Gaussian-shaped anomalies in the stream function field of a two-layer ocean at rest. Bottom topography consisted of a meridional ridge of constant height in the middle of the quadratic model domain. The vertical ring structure, the initial ring position, and the height of the ridge were varied. The general northwestward movement of the model eddies has been shown to be modified toward a more equatorward direction by encountering the upslope of the ridge. Sufficient topographic heights and strong slopes can even block the eddies and force them toward a pure meridional movement. During their translation the eddies lose their vertical coherence. After about 150 days the eddy can only be detected by the surface signal, while the lower layer eddy is dispersed by the radiation of Rossby waves. The passage of "young" (regarding the time between their initialization and their contact with the ridge) and energetic eddies is accompanied by the observation of along-slope currents of significant strength. These may be due to the rectification of radiated Rossby waves at the topographic slope. Only eddies with a significant dynamic signal in the lower layer are influenced by the bottom topography. Strong, shallow eddies over deep lower layers can cross the ridge without strong modification of their translatory movement.
\end{abstract}

\section{Introduction}

The exchange of water between the subtropical gyres of the South Indian and the South Atlantic Oceans is believed to constitute a major component of the global thermohaline circulation [Gordon, 1986]. The leakage from the Indian to the Atlantic comes about in a number of ways, the most important of which is the shedding of large rings by loop occlusion at the retroflection of the Agulhas Current south of Africa [Lutjeharms and Gordon, 1987; Lutjeharms and van Ballegooyen, 1988; Gordon et al., 1992]. This process occurs at irregular intervals [Feron et al., 1992], and the numbers and dimensions of the rings that are shed in this way varies considerably for different periods. A recent review of the dynamics and hydrography of the greater Agulhas Current summarizes what is currently known about this

\footnotetext{
${ }^{1}$ Now at Laboratoire des Ecoulements Géophysiques et Industriels, Institut de Mécanique de Grenoble, Grenoble, France.

${ }^{2}$ Also at Department of Oceanography, University of Cape Town, Rondebosch, South Africa.

Copyright 1999 by the American Geophysical Union.

Paper number 1998JC900127.

0148-0227/99/1998JC900127\$09.00
}

ocean system and the processes involved in ring shedding [Lutjeharms, 1996].

A number of different efforts to model these interbasin exchanges have also been made [e.g., Boudra and Chassignet, 1988; Chassignet and Boudra, 1988; Holland et al., 1991; Thompson et al., 1997; Pichevin et al., 1999; Biastoch and Krauß, 1999], and the successes, and limitations, of these model simulations are discussed in detail by de Ruijter et al. [1999]. A major gap in our understanding that has been identified in both these reviews and that has to date not been adequately addressed is the behavior and ultimate fate of rings in the South Atlantic.

It is known that immediately after having been shed most Agulhas rings move off into the South Atlantic in a broadly northwesterly direction [Duncombe Rae et al., 1989; Duncombe Rae, 1991], thus becoming the major source of mesoscale turbulence in this part of the Southeast Atlantic [Garzoli et al., 1996]. Subsequently, they drift across the full width of the South Atlantic [Gordon and Haxby, 1990; Byrne et al., 1995] slowly losing their initial characteristics. This translational process, partially advective, partially $\beta$-induced [e.g., Nof, 1981], is well simulated in a number of large-scale circulation models [e.g., Lutjeharms and Webb, 1995]. For a proper understanding of the way in which the rings distribute their excess heat and salt in their journey across 
the South Atlantic, a number of things still need to be better understood. These include the rings interaction with the overlying atmosphere and the ambient waters through which they move, the precise routes taken by the rings, as well as their possible interactions with the variable bottom topography along their trajectories.

It has in fact already been recognized that in the region of their inception Agulhas rings are subjected to very large losses of heat to the atmosphere [Walker and $M e y, 1988]$ mainly due to substantial ocean-atmosphere temperature differences there. The season and length of time that they remain close to the Agulhas retroflection, south of $35^{\circ} \mathrm{S}$ latitude, has therefore been shown to have a decided effect on their subsequent hydrographic structure as well as on their overall heat content [Arhan et al., 1999; McDonagh and Heywood, 1999]. Considerable loss of heat leads to convective overturning in these features [e.g., Olson et al., 1992] and consequently a substantial thermostad in older rings; evaporation leads to salinity spiking in surface layers [J.R.E. Lutjeharms and G.M. Rigg, manuscript in preparation, 1999]. The mixing processes between Agulhas rings and South Atlantic waters have to date not been investigated, but hydrographic data suggest a considerable degree of interleaving and double diffusion on the edges of newly formed rings [Olson et al., 1992; Valentine et al., 1988]. Modifications of the trajectories of rings can also be caused by changes in the bottom topography along the paths taken by rings [e.g., Wakker et al., 1990; Byrne et al., 1995].

The normal route taken by Agulhas rings carries them across the Walvis Ridge [Gründlingh, 1995] and the South Atlantic mid-ocean ridge. Altimetric tracking of such rings has suggested that their rate of translation is noticeably reduced on traversing the Walvis Ridge [Gordon and Haxby, 1990; Wakker et al., 1990; Grüdlıngh, 1995]. Byrne et al. [1995] have even suggested that Agulhas rings have a preferential route, crossing more frequently over the deeper parts of the Walvis Ridge than the shallower parts. These possible effects of a shallow ridge on rings of the Agulhas Current should not be a surprise, since the sensitivity of the southern Agulhas Current itself to bottom topography has been well documented [e.g., Lutjeharms and van Ballegooyen, 1984; Matano, 1996].

Kamenkovich et al. [1996] have investigated the effect of the bathymetry on Agulhas rings from a theoretical point of view. They have shown that eddies with the characteristics and dimensions of Agulhas rings do react to crossing a ridge such as the Walvis Ridge. Eddies crossing the ridge exhibit an intensification just before they reach the ridge. This intensification is in the form of a deeper thermocline and a heightened sea surface amplitude. According to their model, the ridge would not markedly affect the transport of heat or salt by the rings, theoretically allowing these anomalous quantities to be carried far into the South Atlantic. The ridge is seen as decelerating eddy propagation. In fact it is only baroclinic eddies that can cross the ridge; barotropic eddies cannot.
In order to improve the understanding of the physical mechanisms responsible for the topographic influences, a series of experiments have been carried out in which not only the degree of baroclinicity of the ring (as given by Kamenkovich et al. [1996]) but also the ridge geometry have been varied. Starting from a flat bottom reference run, the modifications both of the eddy trajectory and its vertical coherence brought about by gradually increasing the height of a meridional ridge are analyzed. This paper is organized as follows: Section 2 presents the numerical quasi-geostrophic model and the experimental configuration. In section 3 the different responses of a baroclinic ring encountering ridges of different heights in an idealized geometry are described. Section 4 presents experiments with eddies of different ages and vertical structures encountering ridges of different width, both in idealized and more realistic model geometries.

\section{Model Configuration}

We use a two-level quasi-geostrophic spectral model with periodic boundary conditions in the horizontal (see Beckmann [1988] for a detailed description). The model solves the well-known vorticity equation

$$
\frac{\partial q}{\partial t}+J(\psi, q)=D
$$

where

$$
q=\nabla^{2} \psi+f_{0}+\beta y+\frac{\partial}{\partial z}\left(\frac{f_{0}^{2}}{N^{2}} \frac{\partial \psi}{\partial z}\right)
$$

Here $\psi$ is the stream function, $f_{0}$ is the Coriolis parameter in the center of the model domain, $\beta$ is the linearized ambient vorticity gradient, $N$ is the BruntVäisälä frequency, $J$ is the Jacobian operator, and $\nabla$ is the horizontal differentiating operator. The dissipation term $D$ is implemented as a biharmonic vorticity proportional friction. Bottom topography appears as a boundary condition for the vertical velocity at the bottom of the lower layer:

$$
w_{B}=J\left(\psi_{2}, h_{B}\right) .
$$

The computational domain is centered at $30^{\circ} \mathrm{S}$ and covers a region of $1280 \mathrm{~km}$ width in both horizontal directions, with a gridspacing of $10 \mathrm{~km}$. In the different experiments, the vertical extent is 1000 and $3500 \mathrm{~m}$, respectively, with an upper layer thickness of $500 \mathrm{~m}$. The time step is chosen to be $4320 \mathrm{~s}$, and the stratification parameter $N=7 \times 10^{-3} \mathrm{~s}^{-1}$ corresponds to observations in the region of the Walvis Ridge [Houry et al., 1987].

The initial eddy shape is a circular Gaussian-shaped extremum in the stream function field. The value of the stream function is chosen to give maximum tangential velocities in the two layers of $0.6 \mathrm{~m} \mathrm{~s}^{-1}$ and $0.2 \mathrm{~m} \mathrm{~s}^{-1}$. The eddy radius is $50 \mathrm{~km}$, which gives a Rossby number $R o=0.17$. Since the model domain is much larger 


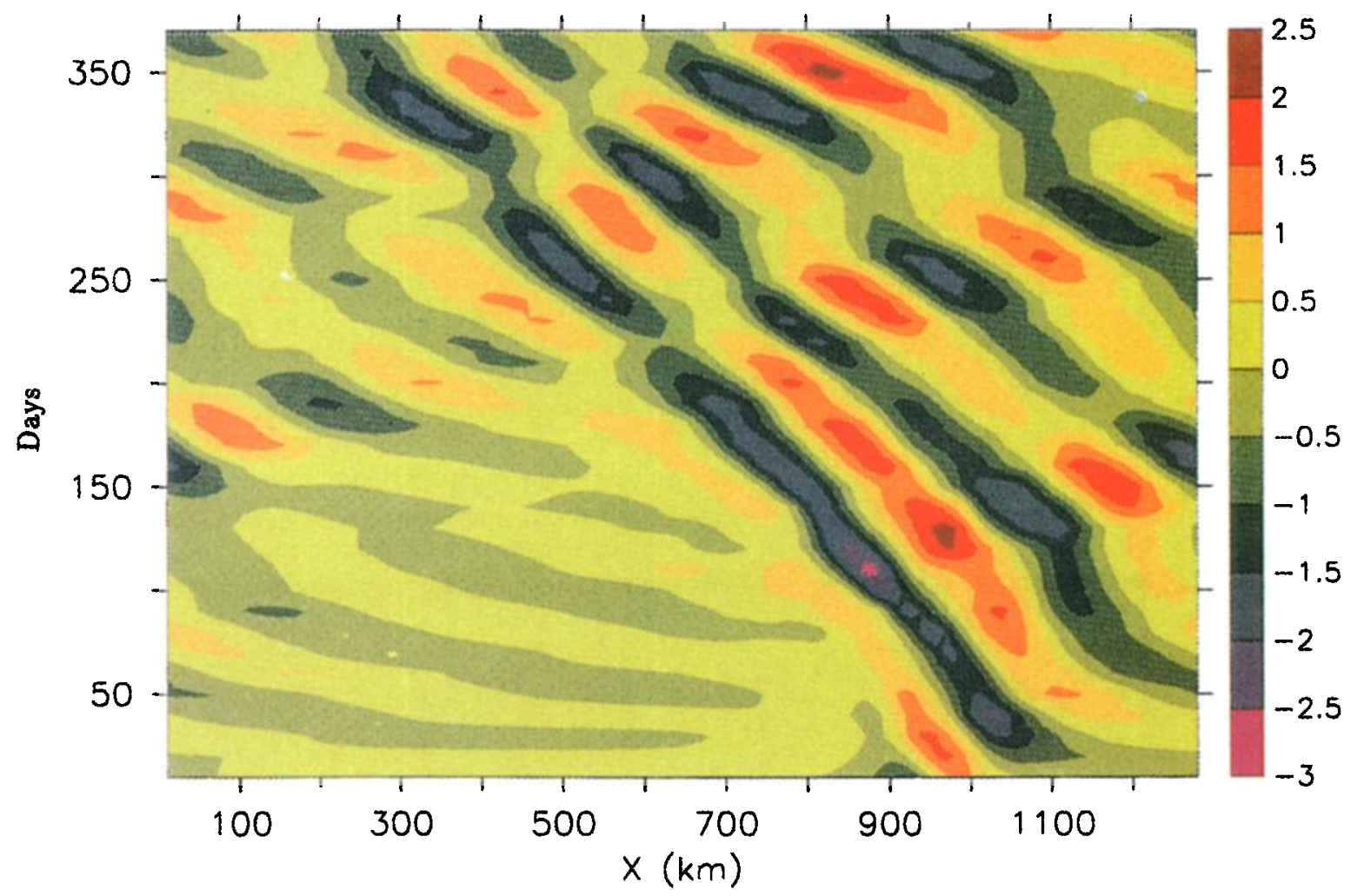

Plate 1. Time evolution of the meridional mean of the lower layer meridional velocity for the flat bottom experiment (in $10 \mathrm{~cm} \mathrm{~s}^{-1}$ ).

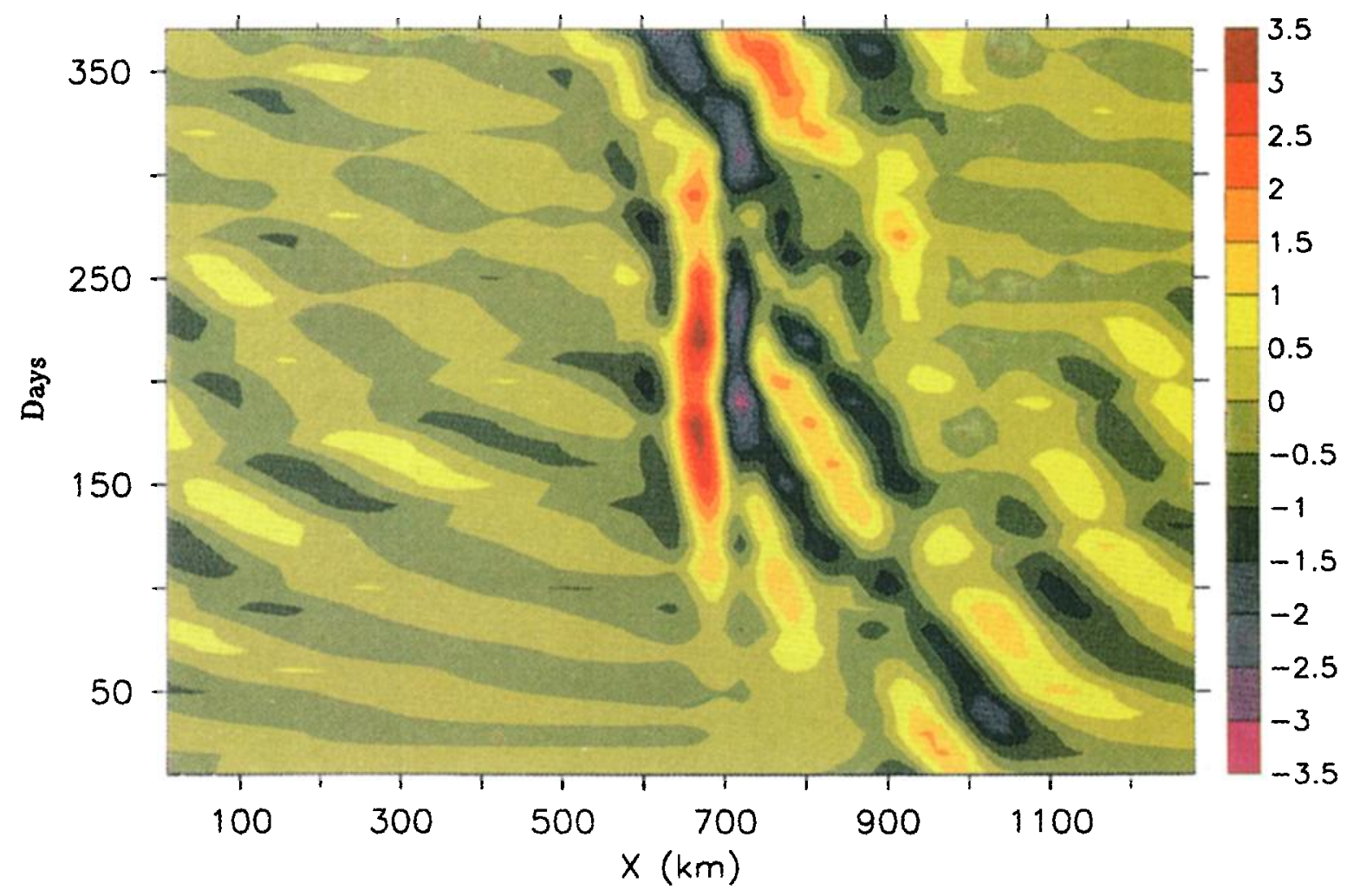

Plate 2. Time evolution of the meridional mean of the lower layer meridional velocity for experiment with a $200 \mathrm{~m}$ ridge (in $10 \mathrm{~cm} \mathrm{~s}^{-1}$ ). The crest of the ridge is at $x=640 \mathrm{~km}$. 


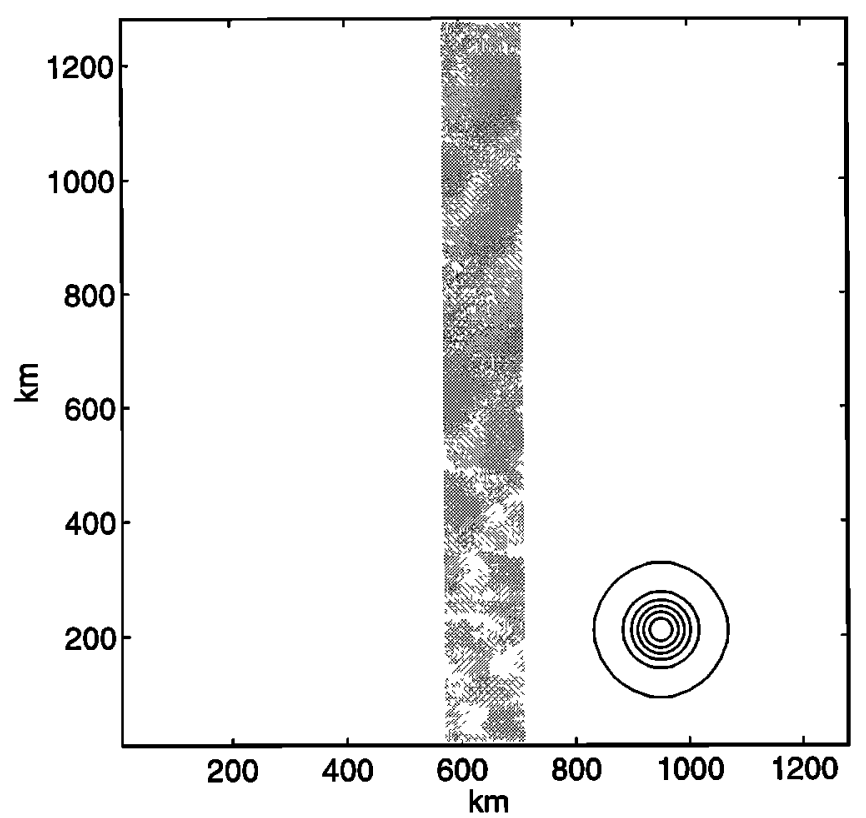

Figure 1. Initial model configuration. The position and $e$-folding width of the meridional ridge are shaded.

than the characteristic scale of the eddy dynamics, we expect no disturbances by the use of periodic boundary conditions. The model is not forced by wind stress nor are any mean currents included.

The topographic obstacle is a meridional ridge of constant height and Gaussian profile. Its width and maximum height are varied in the experiments. Figure 1 shows the model domain with the initial stream function field and the meridional ridge. In order to track the ring path, Lagrangian drifters are placed in the ring center in both layers. These drifters stay close to the ring center during the whole integration period (360 days).

\section{Topographic Influences on Eddy Translation}

We begin our investigation with a series of experiments in a model domain with two equal layers of 500 $m$ thickness and meridional ridges whose height does not exceed $20 \%$ of the total depth. This parameter choice lies well within the validity range of the quasigeostrophic approximation and allows us to identify the effects of bottom topography before proceeding to more realistic model geometries (section 4). Starting from a flat bottom reference experiment, the height of the ridge is increased to 50,100 , and $200 \mathrm{~m}$. The $e$-folding width is $70 \mathrm{~km}$. Figure 2 shows the tracks of the model drifters in the upper layer for three cases. In the flat bottom case the eddy keeps a generally northwestward direction with a mean translation velocity $4 \mathrm{~cm} \mathrm{~s}^{-1}$ (derived from the way traveled during the 1 year integration period). This velocity is at the lower bound of the range of observed translation speeds [e.g., Gordon and Haxby, 1990; Byrne et al., 1995], indicating that advection with mean currents is likely to appear with Agulhas rings.
The experiment with a ridge of $100 \mathrm{~m}$ height clearly shows the influence of the topographic slopes. On encountering the upslope on the eastern side of the rigde after about $\mathbf{4 0}$ days, the ring translation is slowed down, and it takes a more northward direction. It then crosses the ridge in a wide curve, following a more westward course on the downslope and finally continues its movement in a direction parallel to that of the flat bottom experiment. Since the translation velocity is higher during the crossing of the ridge, the eddy still has a mean velocity of $3.5 \mathrm{~cm} \mathrm{~s}^{-1}$.

Increasing the ridge's height to $200 \mathrm{~m}$ changes the path indicated by the drifter dramatically. After about 30 days the eddy switches to a purely meridional movement along the slope of the topographic obstacle. The mean translation speed decreases significantly, and the ring undergoes periods of stagnation, as indicated by slight excursions from the northward pathway. After 230 days the eddy crosses the ridge in a west-northwestward direction.

In in the flat bottom case, the ring's translation is governed by the planetary $\beta$ effect and the force induced by the nonlinearity of the motion; but increasing the height (and thereby the steepness) of the bottom topography augments the influence of the topographic $\beta_{T}\left(\beta_{T}=f_{0} / H \nabla_{h} H\right)$ on the direction of ring translation. The mean $\beta_{T}$ over the upslope of the $200 \mathrm{~m}$ ridge is $8 \times 10^{-11}(\mathrm{~m} \mathrm{~s})^{-1}$, and it reaches a value of 12 $\times 10^{-11}(\mathrm{~m} \mathrm{~s})^{-1}$ at the steepest part of the slope. Since it is significantly greater than the planetary vorticity gradient, the translatory movement above the upslope is strongly controlled by the bottom topography, and the eddy tends toward an equatorward direction.

In contrast to these observations, drifters placed in the center of the lower layer eddy rapidly lose contact

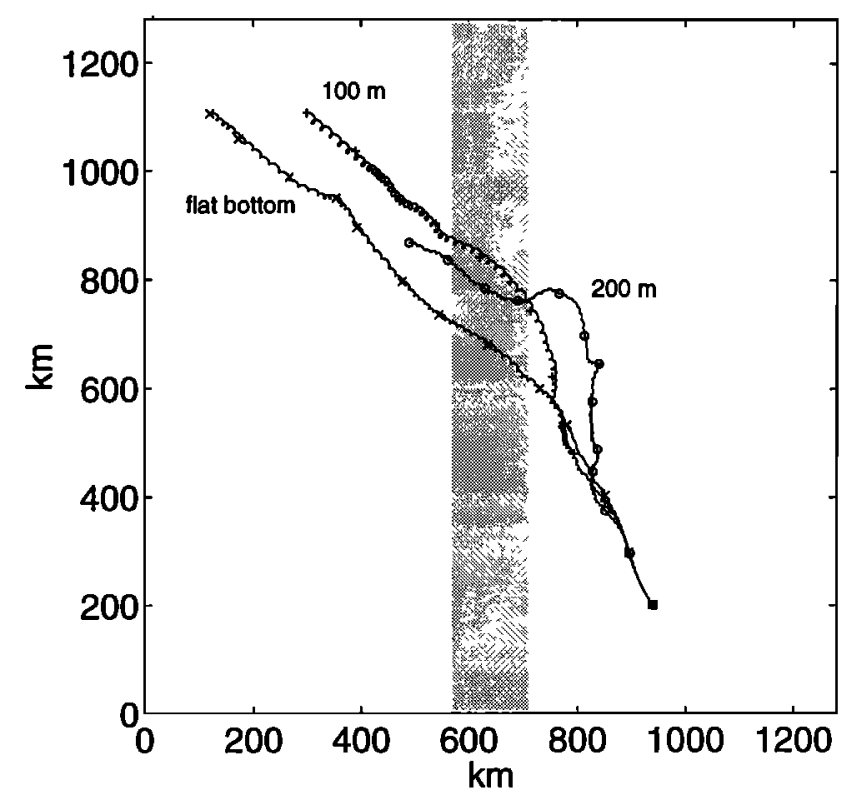

Figure 2. Tracks of drifters in the upper layer eddy center for different heights of the meridional ridge. Time marks are shown every 30 days. The $e$-folding width of the ridge is shaded. 
with the eddy and are advected by the field of radiated waves. Instead of the drifter, tracks we therefore use meridional velocities to study the processes in the lower layer. In spite of the radiation of Rossby waves the passage of the ring itself gives the strongest velocity signal at a given point of the model domain. Because of this, statistics such as the meridional velocity averaged in the meridional direction retain all the information about the ring's passage without being unduly influenced by processes occurring at the edge of the model domain.

For the flat bottom experiment, the temporal evolution of the meridional mean of the lower layer meridional velocity is shown in Plate 1 . The initial vortex position is at $940 \mathrm{~km}$ on the zonal axis. Since the initial eddy stream function maximum is only one third of the value in the upper layer, nonlinearity cannot protect the eddy from dispersion caused by the different phase velocities of the Rossby wave components forming the eddy. The vortex rapidly disperses, and the motion is governed by radiated Rossby waves traveling to the west of the model domain.

In the flat bottom case this westward propagation is uninhibited, but the meridional ridge forms a barrier for these waves in the lower layer in the experiment with topography. As shown in Plate 2, the western half of the model basin remains nearly unaffected by the motion incited by the passage of the ring. An interesting feature of Plate 2 is the appearance of strong meridional velocities on the eastern flank of the ridge, i.e., east of $x=640 \mathrm{~km}$. The radiated Rossby waves seem to rectify at the topographic obstacle and form a pattern of northward and southward velocity bands which persists for more than 100 days. The extrema of these velocities lie in the range of the initial extremum velocities in the lower layer. Comparison with Figure 2 shows that the appearance of this velocity bands coincides with the

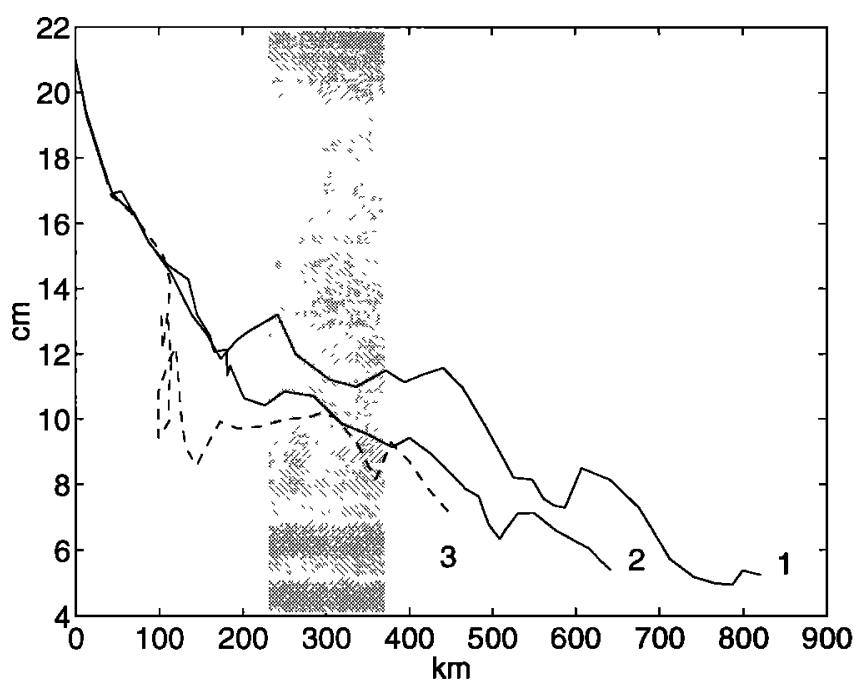

Figure 3. Sea surface elevation versus zonal distance of the eddy center from its initial position. The flat bottom (line 1), $100 \mathrm{~m}$ ridge (line 2 ), and $200 \mathrm{~m}$ ridge (line 3 ) cases are shown. The $e$-folding width of the ridge is shaded.

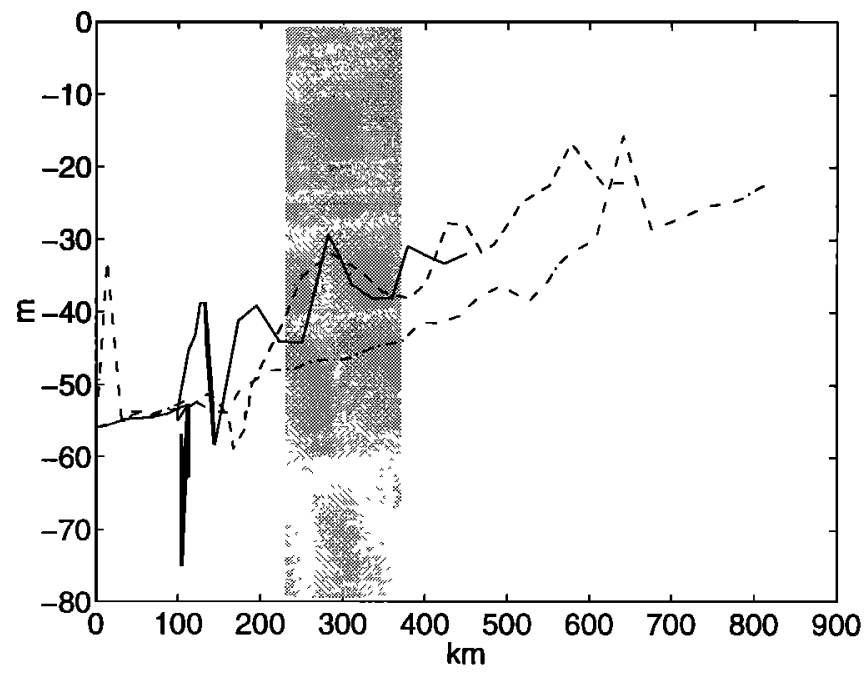

Figure 4. Interface deflection versus zonal distance of the eddy center from its initial position. The flat bottom (dash-dotted line), $100 \mathrm{~m}$ ridge (dashed line), and $200 \mathrm{~m}$ ridge (solid line) cases are shown. The position and $e$-folding width of the meridional ridge are shaded.

phase of purely meridional and slower ring movement. We therefore conclude that the upper layer vortex might be guided by along-slope currents of significant strength in the lower layer.

The evolution of the eddy amplitude (measured by the sea surface elevation, (Figure 3 ) is only slightly affected by the presence of a topographic obstacle, and the values of sea surface elevation after 360 days are very close to each other in the flat and ridge cases, clearly indicating a first-order dependence of eddy amplitude on the time the eddy traveled. For the flat bottom case an exponential zonal decay scale of $1000 \mathrm{~km}$ approximately fits the model results. This zonal distance corresponds to an along track decay scale of about 1460 $\mathrm{km}$, which is lower than the value of $2600 \mathrm{~km}$ reported by Gründlingh et al. [1995] in an analysis of TopexPoseidon altimetric data but close to that of $1700 \mathrm{~km}$ derived from combined Geosat and hydrographic data [Byrne et al., 1995].

The deflection of the layer interface, however, shows an intensification of the vortex when approaching the upslope (Figure 4). Kamenkovich et al. [1996, Figure 19] find the same effect of "thermocline deepening" and a variation of the interface depth after the crossing. After the eddies have reached the western basin, the interface deflection is about $10 \mathrm{~m}$ less than in the flat bottom case, which shows the modification of their vertical structure induced by the ridge.

\section{Influence of Eddy Shape and Age}

We now present some experiments with a ridge of a constant height of $150 \mathrm{~m}$ and eddies initialized at different zonal distances from the ridge. The first ring starts at the same position as in section 3 , i.e., $340 \mathrm{~km}$ east of the crest of the ridge, while in the following experiments this distance is $420 \mathrm{~km}$ and $500 \mathrm{~km}$ (Fig- 


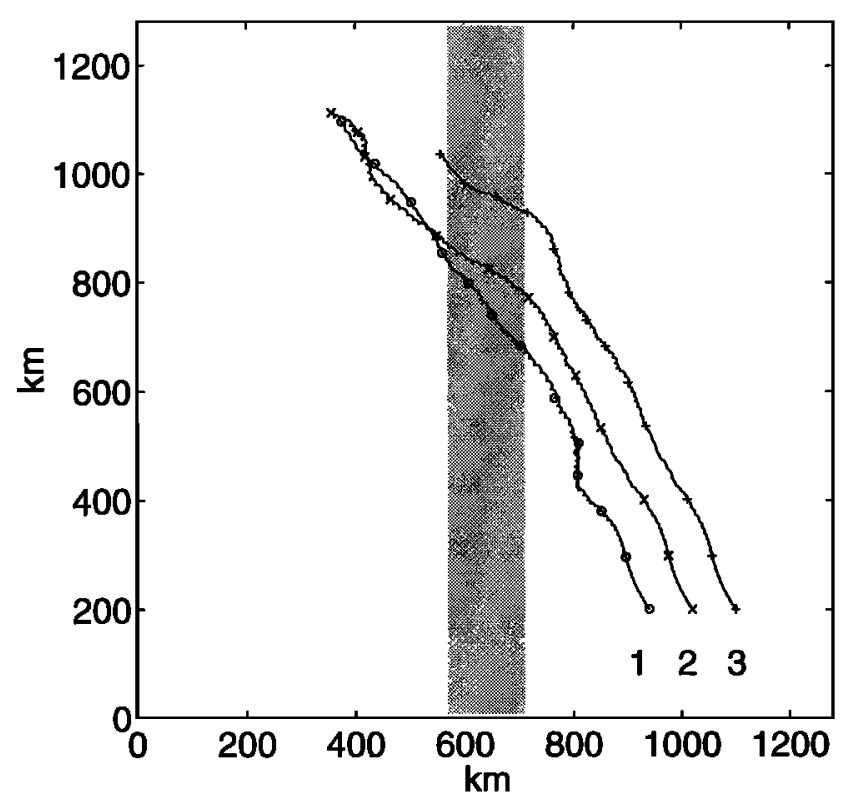

Figure 5. Tracks of drifters in the upper layer eddy center for different initial eddy positions. Time marks are shown every 30 days. The $e$-folding width of the $150 \mathrm{~m}$ high ridge is shaded.

ure 5). While in the first case the vortex is temporarily blocked at the slope of the ridge, the other eddies can cross the ridge with only a slight modification of their path and without significant diminution of their mean speed. Figure 6 shows the stream function field in both layers for experiment 3 of Figure 5, taken as a snapshot before the eddy reaches the slope. The upper layer vortex core is still distinguishable as a coherent feature, whereas the lower layer is dominated by wave-like motion in the wake of the eddy. The upper layer ring has nearly reached the so-called status of "deep compensation" [McWilliams and Flierl, 1979]; that is, there is no motion in phase with the upper layer in the lower layer, and the ring propagation appears to be independent of the lower layer. The nearly independent eddy propagation is even clearer if the eddy is initialized over a motionless lower layer. Figure 7 shows drifter tracks from the flat bottom experiment from section 3 (Figure 2) and an experiment with a $200 \mathrm{~m}$ ridge and a lower layer initially at rest. In the corresponding experiment of section 3 the topographic obstacle was able to block the vortex from crossing. In spite of the presence of bottom topography, the surface intensified vortex takes approximately the same path that the vortex reaching into the lower layer took in the flat bottom case, although it does not reach the same final position.

As a summary of our experiments, Figure 8 shows the mean ring translation velocity (derived from drifter positions) as a function of the height of bottom topography for different vertical ring structures. Calculating a mean velocity over the whole integration period takes away the information about stagnation or acceleration of the vortex, but it allows for comparison the overall effect of bottom topography. For an eddy over a lower layer at rest (line 1 ) the presence of a $50 \mathrm{~m}$ ridge introduces a diminution of the mean velocity of $0.8 \mathrm{~cm} \mathrm{~s}^{-1}$, but the velocity remains nearly unaffected by heightening the ridge (due to the restrictions of the quasigeostrophic approximation, we do not consider topographic obstacles higher than $20 \%$ of the water depth). The same holds true for the upper part of an initially

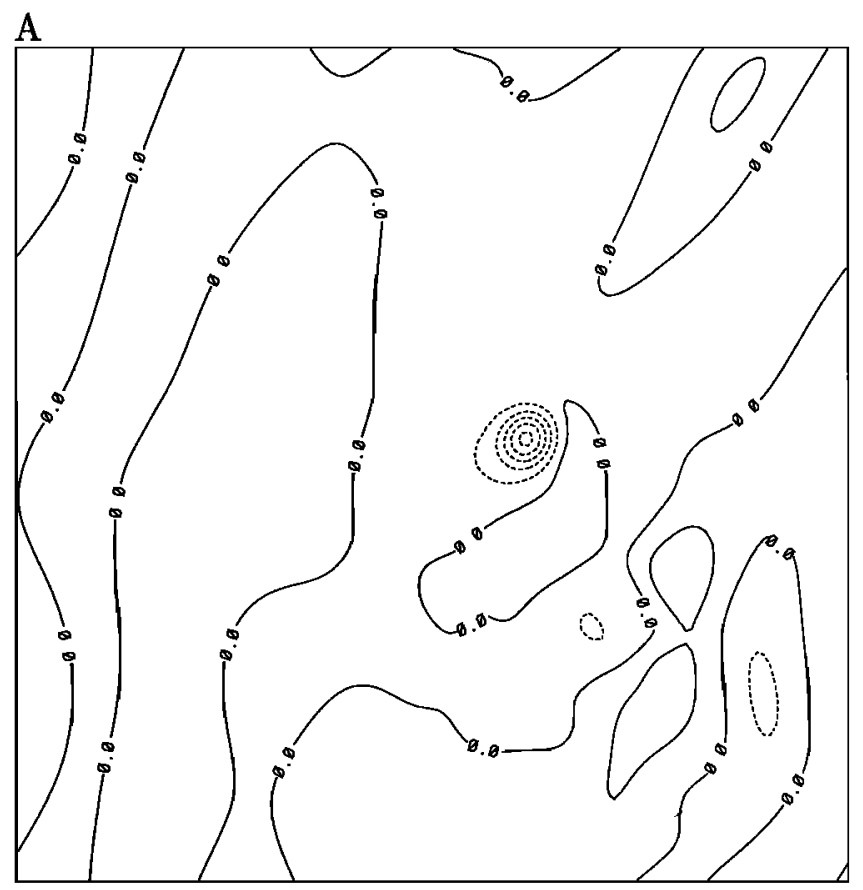

B

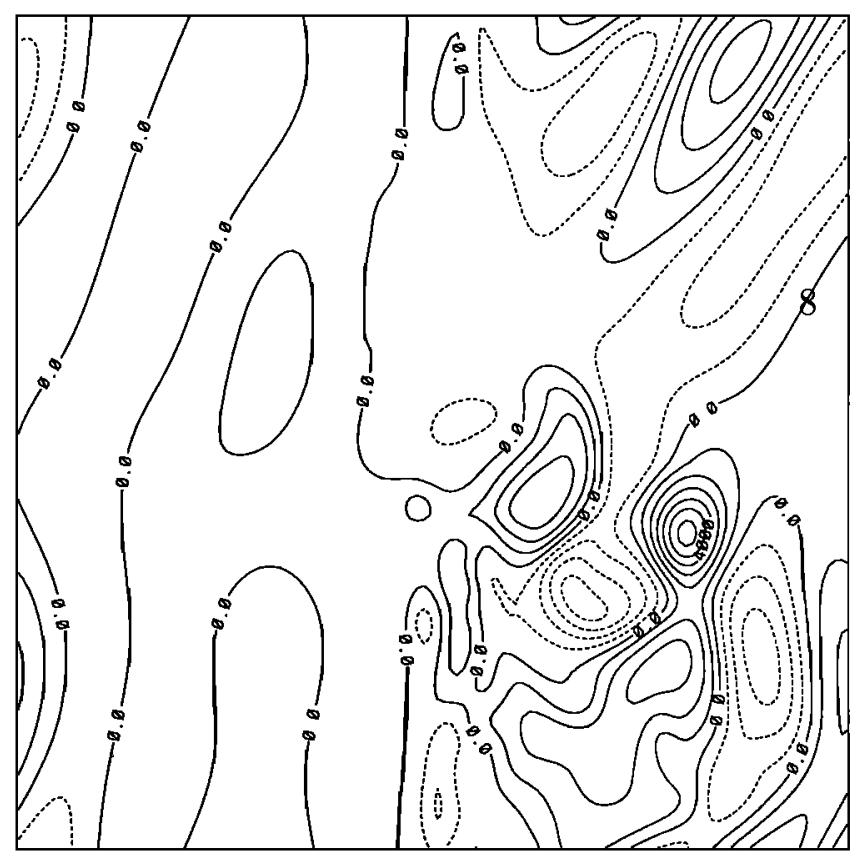

Figure 6. Streamfunction on day 140 of experiment 3 (Figure 7). (A) upper layer, contour interval 3000 $m^{2} s^{-1}$, and (B) lower layer, contour interval 1000 $m^{2} s^{-1}$, are shown. Negative values are dashed lines. 


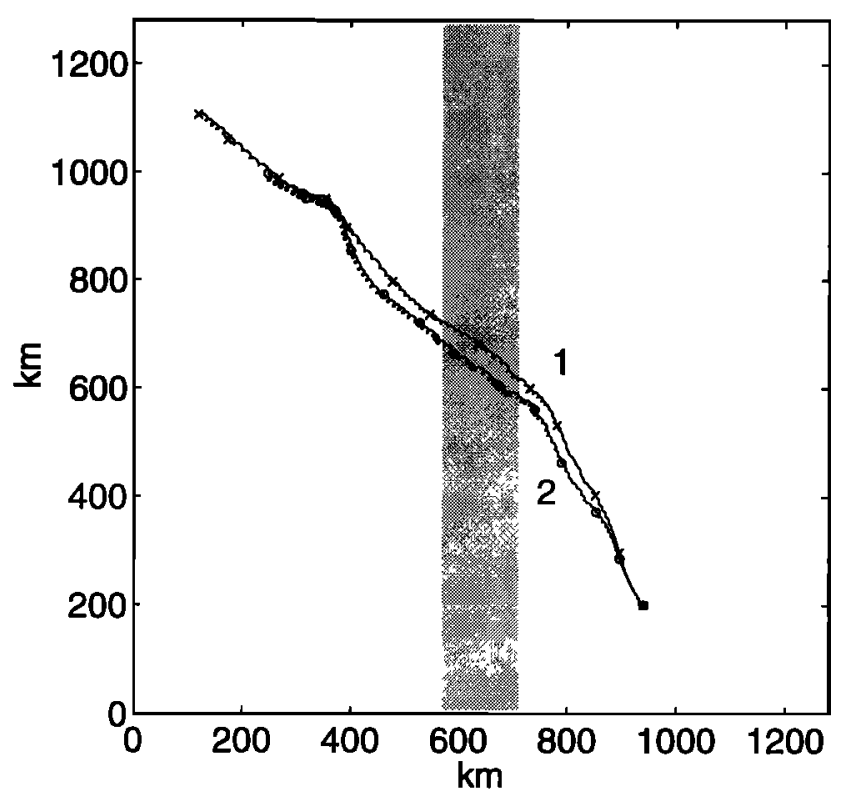

Figure 7. Tracks of drifters in the upper layer eddy center for the flat bottom experiment from Figure 2 (line 1) and an eddy over an initially resting lower layer with a $200 \mathrm{~m}$ ridge (line 2). Time marks are shown every 30 days. The $e$-folding width of the $200 \mathrm{~m}$ high ridge is shaded.

barotropic vortex (line 2), although the diminution of the mean speed is greater $\left(1.3 \mathrm{~cm} \mathrm{~s}^{-1}\right)$. However, a ring in the lower layer with the upper layer at rest clearly feels the growing influence of a higher topographic obstacle. The same diagram but only for the zonal velocity component (Figure 9) shows that the zonal propagation of this lower layer ring is nearly blocked even by a ridge of $50 \mathrm{~m}$ height, whereas, in the other cases, a zonal velocity component can be conserved, although of

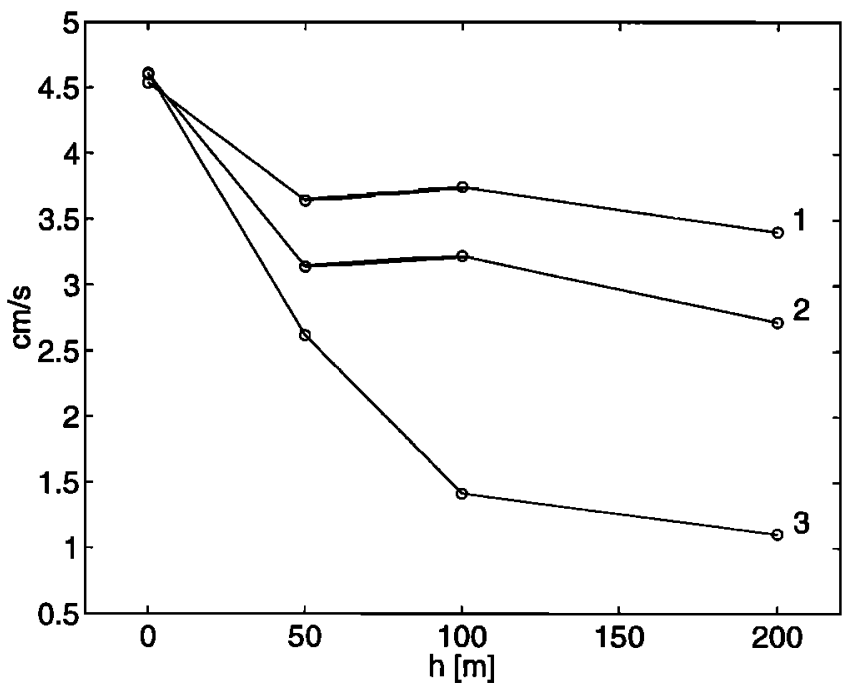

Figure 8. Mean eddy propagation velocity versus height of bottom topography. Surface intensified eddy, lower layer at rest (line 1), barotropic eddy (line 2), and bottom intensified eddy, upper layer at rest (line 3 ) are shown.

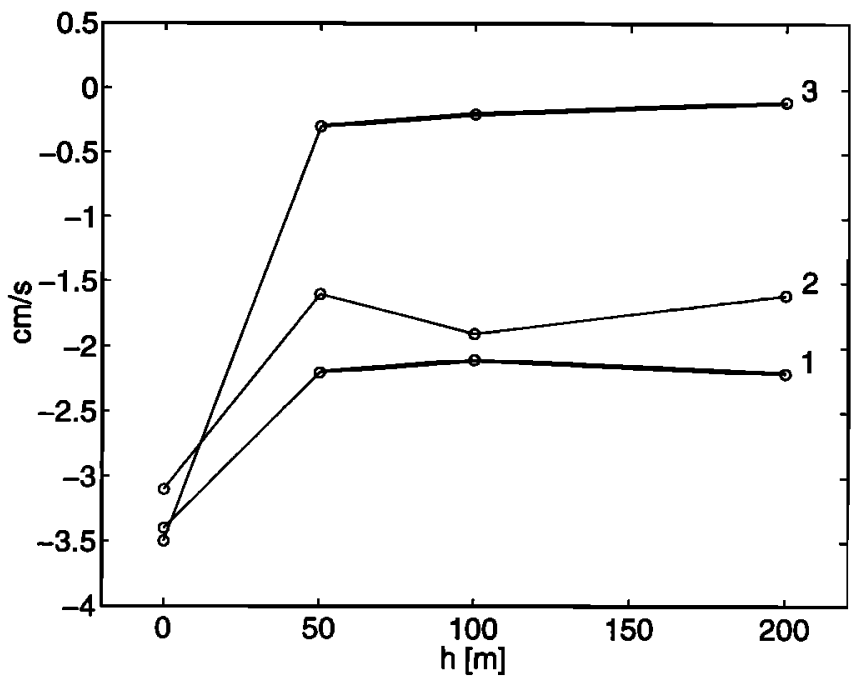

Figure 9. Same as in Figure 10, but for the zonal velocity component.

a lower level than in the flat bottom experiment. The diminution of the propagation speed of eddies crossing the model ridge corresponds qualitatively to the deceleration of eddies crossing the Walvis Ridge as exhibited by Geosat data [Byrne et al., 1995].

We conclude this section with a series of experiments with a lower layer of $3000 \mathrm{~m}$ thickness and a ridge of $700 \mathrm{~m}$ height. The initial eddy radius is $80 \mathrm{~km}$, and the initial maximum tangential velocity is $0.5 \mathrm{~m} \mathrm{~s}^{-1}$ in the upper layer, corresponding to a surface elevation of 28 $\mathrm{cm}$, and $0.21 \mathrm{~m} \mathrm{~s}^{-1}$ in the lower layer. With a $\beta$ Rossby number of $R_{\beta}=3.9$ and a Burger number of $B u=0.38$, these vortices are closer to the parameters observed with Agulhas rings [Kamenkovich et al., 1996]. Trajectory 1 in Figure 10 shows the reference experiment with a flat model domain. As in the previous simulations, the drifter in the center of the upper layer ring shows the northwestward propagation of the anticyclonic vortex.

An eddy encountering the ridge ( $e$-folding width 140 $\mathrm{km}$ ) is slightly decelerated and takes a more westward course to cross the ridge (trajectory 3 ). The stagnation phase (days 30 to 60 ) on the upslope is even clearer for a vortex initialized closer to the ridge (trajectory 4). These two eddies have not switched to the "deep compensation" status before reaching the slope, and therefore both their propagation speed and direction are strongly influenced by the meridional ridge. This leads to a vertical separation of the intially coherent ring, allowing the upper layer ring to cross the ridge.

For experiments 3 and 4 , the process of separation between the two layers is clearly seen from the lower layer drifter tracks in Figure 11. After the stagnation of the upper layer eddy core, the drifters in the lower layer do not follow the westward translation but appear to be completely independent. Again, their mean propagation seems to indicate northward velocities on the eastern side of the ridge.

In the case with a narrower ridge (line 2), the eddy travels during a longer period over flat bottom, allowing for accomplishment of its process of vertical reorgani- 


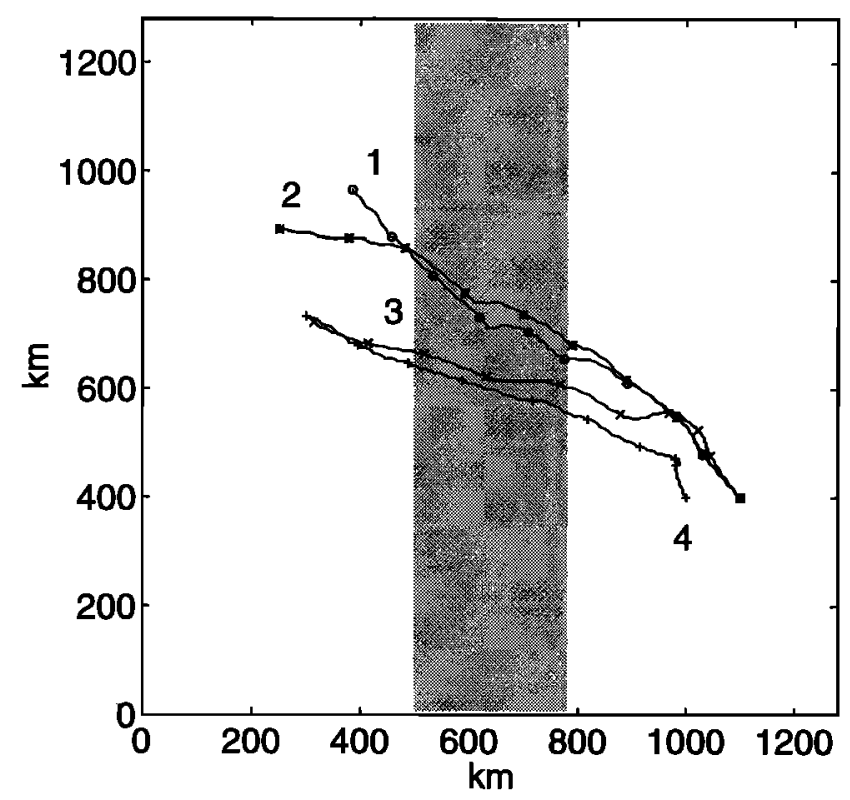

Figure 10. Tracks of drifters in the upper layer eddy center for the experiments with layer thicknesses 500 and $3000 \mathrm{~m}$. The flat bottom (line 1), ridge of $700 \mathrm{~m}$ height and $70 \mathrm{~km}$ width (line 2), ridge of $700 \mathrm{~m}$ height and $140 \mathrm{~km}$ width (line 3 ), and ridge with same measurements as line 3 but different initial position (line 4) cases are shown. Time marks are shown every 30 days. The $140 \mathrm{~km} e$-folding width of the ridge is shaded.

zation. Its path appears to be only slightly modified during the crossing of the ridge, which gives evidence for its being independent of the processes in the lower layer. Furthermore, its initial dynamic signal in the lower layer seems to be too weak to provide for a sufficiently strong along-slope current by rectification of waves radiated from the ring.

\section{Conclusions}

The influence of a meridional ridge on the propagation of isolated vortices in a two-layer ocean on the Southern Hemisphere $\beta$ plane has been investigated by a series of experiments with a numerical quasigeostrophic model. Geographical and dynamical parameters typical for Agulhas rings have been chosen, taking into account the constraints of the quasi-geostrophic approximation. Gaussian shaped vortices were initialized, the vertical structure and starting position of which were varied.

Experiments with two layers of equal thickness revealed the modification of the northwestward translation toward a more equatorward direction. While low ridges do not influence the propagation speed, higher ridges ( $20 \%$ of the total depth) decelerate the eddies and can even force them to move meridionally along the upslope. Along with this purely northward movement of the upper layer vortex, persistent along-slope currents of a significant amplitude were observed in the lower layer. These are believed to be rectificated
Rossby waves which could carry the surface intensified ring along with them.

The intensity of the rings (described by the surface elevation and the interface deflection) depend rather on the distance traveled than on the presence of topographic obstacles. In the ridge cases, the evolution of the interface deflection shows the modification of the vertical eddy structure, whereas the surface elevation does not exhibit the encountering of the ridge, in contrary to the studies of other authors [Kamenkovich et al., 1996]. The time evolution of the surface elevation can be approximated by an exponential function with a decay scale of $1460 \mathrm{~km}$, which is at the lower bound of the decay scales derived from altimetric data [Byrne et al., 1995].

Eddies reaching the ridge in a status of deep compensation, i.e., without a lower layer motion in phase with the upper layer, can cross the topographic obstacle with practically no modification of their propagation. We have shown that besides the vertical coherence a sufficient dynamical signal in the lower layer must be present in order for the ring to feel the influence of bottom topography. Experiments with different layer thicknesses and parameters closer to observed Agulhas rings demonstrated that even shallow, surface intensified vortices can be decelerated by meridional ridge, but translation is resumed after a reorganization of the eddies' vertical structure, and the vortices cross the ridge without further influences.

Our model eddies did not show the vertical coherence that was shown by Kamenkovich et al. [1996] in experiments with a primitive equation model. Nevertheless, the model experiments provide a highly idealized

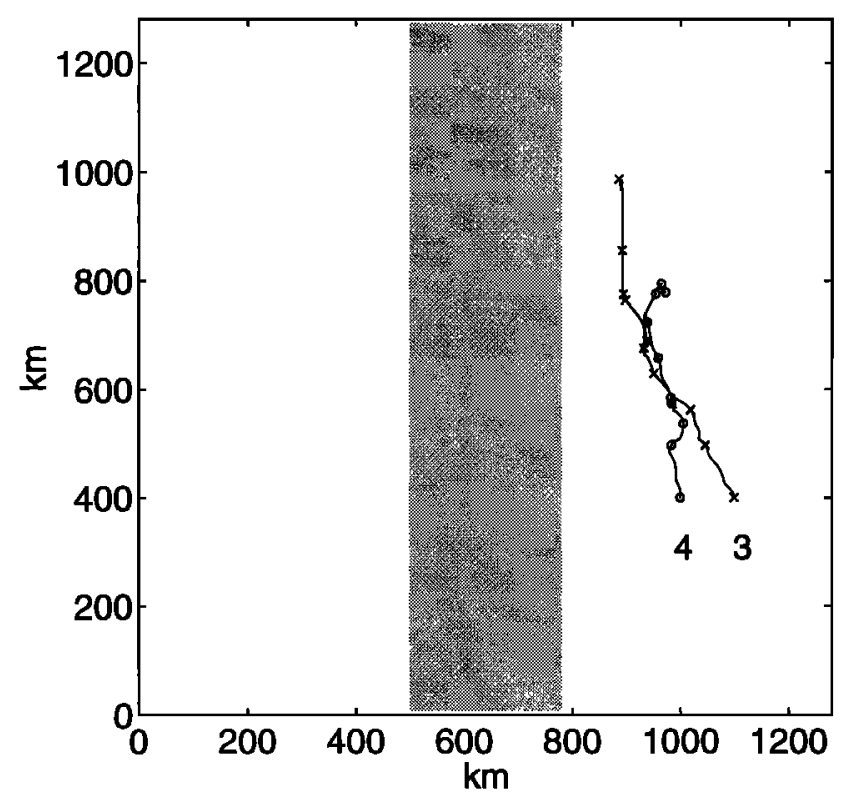

Figure 11. Tracks of drifters in the lower layer eddy center for the experiments with layer thicknesses 500 and $3000 \mathrm{~m}$. For line number explications, see Figure 10. Time marks are shown every 30 days. The $140 \mathrm{~km}$ $e$-folding width of the ridge is shaded. 
study of the behavior of oceanic eddies in the vicinity of topographic obstacles. Owing to the restrictions of the quasigeostrophic approximation and the two-layer approach, it is difficult to represent both topographic features (like the Walvis Ridge, for example) and the vertical structure of an Agulhas ring in a realistic way. The observed effects of Rossby wave radiation and rectification may give some hints for the explanation of a possible topographic control of the translatory movement of these eddies in the South Atlantic Ocean which need to be verified by deep-reaching hydrographic observations.

Future modeling work should focus on the interaction between Agulhas rings and realistically shaped ridges. Byrne et al. [1995] state that Agulhas rings preferably cross the Walvis Ridge at its deepest parts, which seems possible for rings which are sensitive to bottom topography and stagnate on the upslope of a ridge. Therefore, the effect of a ridge of unequal depth should be investigated with a less constraining model and realistic parameters. Furthermore, the dependence of the translation velocity on the steepness of the ridge indicated by drifter data [Olson and Evans, 1986] needs to be systematically clarified.

Acknowledgments. We are grateful to Aike Beckmann for his help in running the model. JOB is supported by the German Academic Exchange Service (DAAD, Doktorandenstipendium HSP III). We thank Alexander von HumboldtStiftung for the award of the Alexander von Humboldt Prize to JREL that made his stay at the Institut für Meereskunde and this collaborative work possible. The South African Foundation for Research Development and the University of Cape Town are thanked for partial financial support.

\section{References}

Arhan, M., H. Mercier, and J. R. E. Lutjeharms, The disparate evolution of three Agulhas rings in the South Atlantic Ocean, J. Geophys. Res., in press, 1999.

Beckmann, A., Vertical structure of midlatitude mesoscale instabilities, J. Phys. Oceanogr., 18, 1354-1371, 1988.

Biastoch, A., and W. Krauß, The role of mesoscale eddies in the source regions of the Agulhas Current, J. Phys. Oceanogr., in press, 1999.

Boudra, D. B., and E. P. Chassignet, Dynamics of Agulhas retroflection and ring formation in a numerical model, I, The vorticity balance, J. Phys. Oceanogr., 18, 280-303, 1988.

Byrne, D. A., A. L. Gordon, and W. F. Haxby, Agulhas eddies: A synoptic view using Geosat ERM data, J. Phys. Oceanogr., 25, 902-917, 1995.

Chassignet, E. P., and D. B. Boudra, Dynamics of Agulhas retroflection and ring formation in a numerical model, II, Energetics and ring formation, J. Phys. Oceanogr., 18, 304-319, 1988.

de Ruijter, W. P. M., J. R. E. Lutjeharms, R. P. Matano, T. Pichevin, A. Biastoch, P. J. van Leeuwen, S. Drijfhout, and W. Weijer, Dynamics, estimation and impact of South Atlantic inter-ocean exchanges, J. Geophys. Res., in press, 1999.

Duncombe Rae, C. M., Agulhas retroflection rings in the South Atlantic Ocean: An overview, S. Afr. J. Mar. Sci., 11, 327-344, 1991.
Duncombe Rae, C. M., L. V. Shannon, and F. A. Shillington, An Agulhas ring in the South Atlantic Ocean, S. Afr. J. Sci., 85, 747-748, 1989.

Feron, R. C. V., W. P. M de Ruijter, and D. Oskam, Ring shedding in the Agulhas Current system, J. Geophys. Res., 97, 9467-9477, 1992.

Garzoli, S. L., A. L. Gordon, V. Kamenkovich, D. Pillsbury, and C. Duncombe Rae, Variability and sources of the southeastern Atlantic circulation, J. Mar. Res., 54, 1039-1071, 1996.

Gordon, A. L., Interocean exchange of thermocline water, J. Geophys. Res., 91, 5037-5046, 1986.

Gordon, A. L., and W. F Haxby, Agulhas eddies invade the South Atlantic: Evidence from Geosat altimeter and shipboard conductivity-temperature-depth survey, J. Geophys. Res., 95, 2991-3006, 1990.

Gordon, A. L., R. F. Weiss, W. M. Smethie, and M. J. Warner, Thermocline and intermediate water communication between the South Atlantic and Indian Oceans, $J$. Geophys. Res., 97, 7223-7240, 1992.

Gründlingh, M. L., Tracking eddies in the southeast Atlantic and southwest Indian oceans with TOPEX/POSEIDON, J. Geophys. Res., 100, 24977-24986, 1995.

Holland, W. R., V. Zlotnicki, and L.-L. Fu, Modelled timedependent flow in the Agulhas retroflection region as deduced from altimeter data assimilation, S. Afr. J. Mar. Sci., 10, 407-427, 1991.

Houry, S., E. Dombrowsky, P. De Mey, and J.-F. Minster, Brunt-Väisälä-Frequency and Rossby radii in the South Atlantic, J. Phys. Oceanogr., 17, 1619-1626, 1987.

Kamenkovich, V. M., Y.P. Leonov, D.A. Nechaev, D.A. Byrne, and A.L. Gordon, On the influence of bottom topography on the Agulhas eddy, J. Phys, Oceanogr., 26, 892-912, 1996.

Lutjeharms, J. R. E., The exchange of water between the South Indian and South Atlantic Oceans, in The South Atlantic: Present and Past Circulation, edited by G. Wefer et al., pp. 125-162, Springer Verlag, New York, 1996.

Lutjeharms, J. R. E., and A. L. Gordon, Shedding of an Agulhas ring observed at sea, Nature, 325, 138-140, 1987.

Lutjeharms, J. R. E., and R. C. van Ballegooyen, Topographic control in the Agulhas Current system, Deep Sea Res. Part I, 31, 1321-1337, 1984.

Lutjeharms, J. R. E., and R. C. van Ballegooyen, The Agulhas Current retroflection, J. Phys. Oceanogr., 18, 15701583, 1988.

Lutjeharms, J. R. E., and D. J. Webb, Modelling the Agulhas Current system with FRAM (Fine Resolution Antarctic Model), Deep Sea Res. Part I, 42, 523-551, 1995.

Matano, R. P., A numerical study of the Agulhas retroflection: The role of bottom topography, J. Phys. Oceanogr., 26, 2267-2279, 1996.

McDonagh, E. L., and K. J. Heywood, The origin of an anomalous ring in the Southeast Atlantic, J. Phys. Oceanogr., in press, 1999.

McWilliams, J. C., and G. R. Flierl, On the evolution of isolated, nonlinear vortices, J. Phys. Oceanogr., 9, 1155$1182,1979$.

Nof, D., On the $\beta$-induced movement of isolated baroclinic eddies, J. Phys. Oceanogr., 11, 1662-1672, 1981.

Olson, D. B., and R. H. Evans, Rings of the Agulhas Current, Deep Sea Res. Part I, 33, 27-42, 1986.

Olson, D. B., R. A. Fine, and A. L. Gordon, Convective modifications of water masses in the Agulhas, Deep Sea Res. Part I, 39, 5163-5181, 1992.

Pichevin, T., D. Nof, and J. R. E. Lutjeharms, Why are there Agulhas rings?, J. Phys. Oceanogr., in press, 1999.

Thompson, S. R., D. P Stevens, and K. Döös, The impor- 
tance of interocean exchange south of Africa in a numerical model, J. Geophys. Res., 102, 3303-3315, 1997.

Valentine, H. R., C. M. Duncombe Rae, R. C. van Ballegooyen, and J. R. E. Lutjeharms, The Subtropical Convergence and Agulhas Retroflection Cruise (SCARC): Data report, Report T/SEA 8804, 10 pp., 56 tables, Counc. for Sci. Ind. Res., Pretoria, South Africa, 1988.

Wakker, K. F., R. C. A. Zandbergen, M. C. Naeije, and B. A. C. Ambrosius, Geosat altimeter data analysis for the oceans around South Africa, J. Geophys. Res., 95, 29913006, 1990.

Walker, N. D., and R. D. Mey, Ocean/atmosphere heat fluxes within the Agulhas retroflection region, J. Geophys. Res., 93, 15473-15483, 1988.
J.-O. Beismann, Laboratoire des Ecoulements Géophysiques et Industriels, Institut de Mécanique de Grenoble, BP 53 X, 38041 Grenoble cedex 9, France. (JensOlaf.Beismann@hmg.inpg.fr)

R. H. Käse, and J. R. E. Lutjeharms, Institut für Meereskunde an der Universität Kiel, Düsternbrooker Weg 20, 24105 Kiel, Germany.

(Received March 30, 1998; revised October 6, 1998; accepted December 24, 1998.) 